\title{
The cosmic ray shadow of the Moon observed with the ANTARES neutrino telescope
}

A. Albert ${ }^{1}$, M. André ${ }^{2}$, M. Anghinolfi ${ }^{3}$, G. Anton ${ }^{4}$, M. Ardid ${ }^{5}$, J. -J. Aubert ${ }^{6}$, J. Aublin ${ }^{7}$, T. Avgitas $^{7}$, B. Baret ${ }^{7}$, J. Barrios-Martít ${ }^{8}$, S. Basa ${ }^{9}$, B. Belhorma ${ }^{10}$, V. Bertin ${ }^{6}$, S. Biagi ${ }^{11}$, R. Bormuth ${ }^{12,13}$, J. Boumaaza ${ }^{14}$, S. Bourret ${ }^{7}$, M. C. Bouwhuis ${ }^{12}$, H. Brânzaş ${ }^{15}$, R. Bruijn ${ }^{12,16}$, J. Brunner ${ }^{6}$, J. Busto ${ }^{6}$, A. Capone ${ }^{17,18}$, L. Caramete $^{15}$, J. Carr ${ }^{6}$, S. Celli ${ }^{17,18,19}$, M. Chabab $^{20}$, R. Cherkaoui El Moursli ${ }^{14}$, T. Chiarusi ${ }^{21, a}$, M. Circella ${ }^{22}$, J. A. B. Coelho ${ }^{7}$, A. Coleiro ${ }^{7,8}$, M. Colomer ${ }^{7,8}$, R. Coniglione ${ }^{11}$, H. Costantini ${ }^{6}$, P. Coyle ${ }^{6}$, A. Creusot ${ }^{7}$, A. F. Díaz ${ }^{23}$, A. Deschamps ${ }^{24}$, C. Distefano ${ }^{11}$, I. Di Palma ${ }^{17,18}$, A. Domi ${ }^{3,25}$, C. Donzaud ${ }^{7,26}$, D. Dornic ${ }^{6}$, D. Drouhin ${ }^{1}$, T. Eberl ${ }^{4}$, I. El Bojaddaini ${ }^{27}$, N. El Khayati ${ }^{14}$, D. Elsässer ${ }^{28}$, A. Enzenhöfer ${ }^{4,6}$, A. Ettahiri ${ }^{14}$, F. Fassi ${ }^{14}$, I. Felis ${ }^{5}$, P. Fermani ${ }^{17,18}$, G. Ferrara ${ }^{11}$, L. A. Fusco ${ }^{7,29}$, P. Gay ${ }^{7,30}$, H. Glotin ${ }^{31}$, T. Grégoire ${ }^{7}$, R. Gracia Ruiz ${ }^{1}$, K. Graf ${ }^{4}$, S. Hallmann ${ }^{4}$, H. van Haren ${ }^{32}$, A. J. Heijboer ${ }^{12}$, Y. Hello ${ }^{24}$, J. J. Hernández-Rey ${ }^{8}$, J. Hößl ${ }^{4}$, J. Hofestädt ${ }^{4}$, G. Illuminati ${ }^{8}$, M. de Jong ${ }^{12,13}$, M. Jongen ${ }^{12}$, M. Kadler ${ }^{28}$, O. Kalekin ${ }^{4}$, U. Katz ${ }^{4}$, N. R. Khan-Chowdhury ${ }^{8}$, A. Kouchner ${ }^{7,33}$, M. Kreter $^{28}$, I. Kreykenbohm ${ }^{34}$, V. Kulikovskiy ${ }^{3,35}$, C. Lachaud ${ }^{7}$, R. Lahmann ${ }^{4}$, D. Lefèvre ${ }^{36,37}$, E. Leonora ${ }^{38}$, G. Levi ${ }^{21,29}$, M. Lotze $^{8}$, S. Loucatos ${ }^{7,39}$, M. Marcelin ${ }^{9}$, A. Margiotta ${ }^{21,29}$, A. Marinelli ${ }^{40,41}$, J. A. Martínez-Mora ${ }^{5}$, R. Mele ${ }^{42,43}$, K. Melis ${ }^{12,16}$, P. Migliozzi ${ }^{42}$, A. Moussa ${ }^{27}$, S. Navas ${ }^{44}$, E. Nezri ${ }^{9}$, A. Nuñez ${ }^{6,9}$, M. Organokov ${ }^{1}$, G. E. Păvălaş ${ }^{15}$, C. Pellegrino ${ }^{21,29}$, P. Piattelli ${ }^{11}$, V. Popa ${ }^{15}$, T. Pradier ${ }^{1}$, L. Quinn ${ }^{6}$, C. Racca $^{45}$, N. Randazzo ${ }^{38}$, G. Riccobene $^{11}$, A. Sánchez-Losa ${ }^{22}$, M. Saldaña ${ }^{5}$, I. Salvadori ${ }^{6}$, D. F. E. Samtleben ${ }^{12,13}$, M. Sanguineti ${ }^{3,25, b}$ De, P. Sapienza $^{11}$, F. Schüssler ${ }^{39}$, M. Spurio ${ }^{21,29}$, Th. Stolarczyk ${ }^{39}$, M. Taiuti ${ }^{3,25}$, Y. Tayalati $^{14}$, A. Trovato $^{11}$, B. Vallage $^{7,39}$, V. Van Elewyck ${ }^{7,33}$, F. Versari ${ }^{21,29}$, D. Vivolo ${ }^{42,43}$, J. Wilms ${ }^{34}$, D. Zaborov ${ }^{6}$, J. D. Zornoza ${ }^{8}$ J. Zúñiga $^{8}$

${ }^{1}$ Université de Strasbourg, CNRS, IPHC UMR 7178, 67000 Strasbourg, France

${ }^{2}$ Laboratory of Applied Bioacoustics, Technical University of Catalonia, Rambla Exposició, 08800 Vilanova i la Geltrú, Barcelona, Spain

${ }^{3}$ INFN, Sezione di Genova, Via Dodecaneso 33, 16146 Genova, Italy

${ }^{4}$ Friedrich-Alexander-Universität Erlangen-Nürnberg, Erlangen Centre for Astroparticle Physics, Erwin-Rommel-Str. 1, Erlangen, Germany

${ }^{5}$ Institut d'Investigació per a la Gestió Integrada de les Zones Costaneres (IGIC), Universitat Politècnica de València. C/ Paranimf 1, 46730 Gandia, Spain

${ }^{6}$ Aix Marseille Univ, CNRS/IN2P3, CPPM, Marseille, France

${ }^{7}$ APC, Univ Paris Diderot, CNRS/IN2P3, CEA/Irfu, Obs de Paris, Sorbonne Paris Cité, Paris, France

${ }^{8}$ IFIC, Instituto de Física Corpuscular (CSIC, Universitat de València) c/ Catedrático José Beltrán, 2 E-46980 Paterna, Valencia, Spain

${ }^{9}$ LAM - Laboratoire d'Astrophysique de Marseille, Pôle de l'Étoile Site de Château-Gombert, rue Frédéric Joliot-Curie 38, 13388 Marseille Cedex 13, France

${ }^{10}$ National Center for Energy Sciences and Nuclear Techniques, B.P.1382, 10001 Rabat, Morocco

${ }^{11}$ INFN, Laboratori Nazionali del Sud (LNS), Via S. Sofia 62, 95123 Catania, Italy

12 Nikhef, Science Park, Amsterdam, The Netherlands

${ }^{13}$ Huygens-Kamerlingh Onnes Laboratorium, Universiteit Leiden, Leiden, The Netherlands

${ }^{14}$ Faculty of Sciences, University Mohammed V in Rabat, 4 av. Ibn Battouta, B.P. 1014, 10000 Rabat, Morocco

15 Institute of Space Science, RO-077125 Măgurele, Bucharest, Romania

${ }^{16}$ Universiteit van Amsterdam, Instituut voor Hoge-Energie Fysica, Science Park 105, 1098 XG Amsterdam, The Netherlands

${ }^{17}$ INFN, Sezione di Roma, P.le Aldo Moro 2, 00185 Rome, Italy

18 Dipartimento di Fisica dell’Università La Sapienza, P.le Aldo Moro 2, 00185 Rome, Italy

19 Gran Sasso Science Institute, Viale Francesco Crispi 7, 00167 L'Aquila, Italy

${ }^{20}$ LPHEA, Faculty of Science, Semlali, Cadi Ayyad University, P.O.B. 2390, Marrakech, Morocco

${ }^{21}$ INFN, Sezione di Bologna, Viale Berti-Pichat 6/2, 40127 Bologna, Italy

22 INFN, Sezione di Bari, Via E. Orabona 4, 70126 Bari, Italy

23 Department of Computer Architecture and Technology/CITIC, University of Granada, 18071 Granada, Spain

${ }^{24}$ Géoazur, UCA, CNRS, IRD, Observatoire de la Côte d'Azur, Sophia Antipolis, France

${ }^{25}$ Dipartimento di Fisica dell'Università, Via Dodecaneso 33, 16146 Genova, Italy

${ }^{26}$ Université Paris-Sud, 91405 Orsay Cedex, France

${ }^{27}$ Laboratory of Physics of Matter and Radiations, University Mohammed I, B.P.717, 6000 Oujda, Morocco

28 Institut für Theoretische Physik und Astrophysik, Universität Würzburg, Emil-Fischer Str. 31, 97074 Würzburg, Germany 
${ }^{29}$ Dipartimento di Fisica e Astronomia dell'Università, Viale Berti Pichat 6/2, 40127 Bologna, Italy

${ }^{30}$ Laboratoire de Physique Corpusculaire, Clermont Université, Université Blaise Pascal, CNRS/IN2P3, BP 10448, 63000 Clermont-Ferrand, France

${ }^{31}$ LIS, UMR Université de Toulon, Aix Marseille Université, CNRS, 83041 Toulon, France

32 Royal Netherlands Institute for Sea Research (NIOZ) and Utrecht University, Landsdiep 4, 1797 SZ 't Horntje (Texel), The Netherlands

${ }^{33}$ Institut Universitaire de France, 75005 Paris, France

${ }^{34}$ Dr. Remeis-Sternwarte and ECAP, Friedrich-Alexander-Universität Erlangen-Nürnberg, Sternwartstr. 7, 96049 Bamberg, Germany

${ }^{35}$ Skobeltsyn Institute of Nuclear Physics, Leninskie gory, Moscow State University, 119991 Moscow, Russia

${ }^{36}$ Mediterranean Institute of Oceanography (MIO), Aix-Marseille University, 13288 Marseille, Cedex 9, France

${ }^{37}$ Université du Sud Toulon-Var, CNRS-INSU/IRD UM 110, 83957 La Garde Cedex, France

${ }^{38}$ INFN, Sezione di Catania, Via S. Sofia 64, 95123 Catania, Italy

39 IRFU, CEA, Université Paris-Saclay, 91191 Gif-sur-Yvette, France

40 INFN, Sezione di Pisa, Largo B. Pontecorvo 3, 56127 Pisa, Italy

${ }^{41}$ Dipartimento di Fisica dell'Università, Largo B. Pontecorvo 3, 56127 Pisa, Italy

42 INFN, Sezione di Napoli, Via Cintia, 80126 Napoli, Italy

${ }^{43}$ Dipartimento di Fisica dell'Università Federico II di Napoli, Via Cintia, 80126 Napoli, Italy

${ }^{44}$ Dpto. de Física Teórica y del Cosmos \& C.A.F.P.E., University of Granada, 18071 Granada, Spain

${ }^{45}$ GRPHE, Université de Haute Alsace - Institut universitaire de technologie de Colmar, 34 rue du Grillenbreit, BP 50568, 68008 Colmar, France

Received: 5 August 2018 / Accepted: 14 November 2018 / Published online: 11 December 2018

(C) The Author(s) 2018

\begin{abstract}
One of the main objectives of the ANTARES telescope is the search for point-like neutrino sources. Both the pointing accuracy and the angular resolution of the detector are important in this context and a reliable way to evaluate this performance is needed. In order to measure the pointing accuracy of the detector, one possibility is to study the shadow of the Moon, i.e. the deficit of the atmospheric muon flux from the direction of the Moon induced by the absorption of cosmic rays. Analysing the data taken between 2007 and 2016, the Moon shadow is observed with 3.5 $\sigma$ statistical significance. The detector angular resolution for downwardgoing muons is $0.73^{\circ} \pm 0.14^{\circ}$. The resulting pointing performance is consistent with the expectations. An independent check of the telescope pointing accuracy is realised with the data collected by a shower array detector onboard of a ship temporarily moving around the ANTARES location.
\end{abstract}

\section{Introduction}

The detection of cosmic neutrinos is a new and unique method to study the Universe. The weakly interacting nature of neutrinos makes them a complementary cosmic probe to other messengers such as the electromagnetic radiation, $\gamma$-rays, gravitational waves and charged cosmic rays. Neutrinos can travel cosmological distances, crossing regions with high matter or radiation field densities, without being absorbed. They allow the observation of the distant Universe and the interior of the astrophysical sources.

A milestone has been set with the first evidence of a cosmic signal of high-energy neutrinos [1] by the IceCube detector $[2,3]$. The ANTARES telescope [4], although much smaller

\footnotetext{
a e-mail: tommaso.chiarusi@bo.infn.it

b e-mail: matteo.sanguineti@ge.infn.it
}

than the IceCube detector, is the largest undersea neutrino telescope currently in operation. One of its main goals is the search for astrophysical point-like sources of neutrinos. To this aim, the pointing accuracy of the detector is important and an evaluation of this performance is required.

The interaction of cosmic rays in the atmosphere produces downward-going muons that can be recorded by underground, underice or underwater experiments. Atmospher-ic muons represent a large source of background for cosmic neutrino detection, but at the same time they can be used to calibrate the detector. Due to absorption effects of cosmic rays by the Moon, a deficit in the atmospheric muon event density (expressed as number of events per square degrees) in the direction of the Moon, the so-called Moon shadow, is expected. With this approach, the Moon shadow has been already measured and reported by MACRO [5], SOUDAN [6], L3+Cosmics [7] and by IceCube [8] Collaborations. It is worthy to mention here that other experiments, like CYGNUS [9], TIBET [10], CASA [11], ARGO-YBJ [12], and recently also HAWC [13] measured the Moon shadow by exploiting surface arrays detectors.

This work presents the first measurement of ANTARES angular resolution with atmospheric downward-going muons and the detector pointing performance making use of a celestial source for calibrations. A complementary estimation of the telescope pointing accuracy has been performed by means of a surface array of particle detectors arranged onboard a ship deck. The ship was temporarily routing above the ANTARES detector, allowing to correlate the signals from the detection of atmospheric showers with the signals induced by downward-going muons in the underwater telescope. 
This paper is organized as follows: in Sect. 2 the ANTARES detector is introduced together with the motivations of the present analysis; in Sect. 3 the Moon shadow analysis is described; the surface array analysis is presented in Sect. 4 and the conclusions are reported in Sect. 5.

\section{The ANTARES neutrino telescope}

The ANTARES detector is deployed $40 \mathrm{~km}$ offshore from Toulon, France $\left(42^{\circ} 48^{\prime} \mathrm{N}, 6^{\circ} 10^{\prime} \mathrm{E}\right)$ anchored at a depth of about $2475 \mathrm{~m}$. The telescope detects the Cherenkov light stimulated in the medium by relativistic particles by means of a three dimensional grid of optical modules (OMs), pressure resistant glass spheres each containing one $10^{\prime \prime}$ photomultiplier tube (PMT). The OMs are arranged in triplets, forming a storey, along twelve vertical lines, for a total of 885 OMs [4]. The lines are anchored on the sea bottom and kept taut by a buoy at the top. Each PMT is nominally oriented $45^{\circ}$ downward with respect to the vertical direction. This orientation enhances the efficiency for the reconstruction of upward-going tracks, but still allows the detection of downward-going muons with smaller efficiency. A titanium cylinder in each storey houses the electronics for readout and control, together with compasses and tiltmeters. The total length of each line is $450 \mathrm{~m}$, without any instrument along the lower $100 \mathrm{~m}$. The distance between storeys is 14.5 $\mathrm{m}$ and the distance between two lines ranges between 60 and $75 \mathrm{~m}$. The lines are connected to a central junction box which, in turn, is connected to shore via an electro-optical cable. Due to sea currents, a positioning system comprising hydrophones, compasses and tiltmeters is used to monitor the detector geometry [14]. Finally the absolute orientation is provided by the triangulation of acoustic signals between lines and the deployment vessel at the sea surface using GPS $[14,15]$. The first detection line was deployed in 2006; the detector was completed in 2008.

The recorded information of each photon detected on a PMT is referred to as hit, and consists of the detection time, the amount of electric charge measured on the PMT anode and the PMT identification. The ensemble of hits contained in a certain time-window, identified after some trigger condition, is called event. Muon candidates are identified by requiring spacetime causality between the hits of one event $[16,17]$. The quality of the reconstruction of muon trajectories depends on the goodness of such spacetime correlation.

\section{The Moon shadow analysis}

Atmospheric muons are a valuable resource for validating the detector performance and characterising some of the possible systematics associated to the experimental setup. The muons produced in the interactions of primary cosmic rays in the upper layers of the atmosphere can traverse several kilometres of water equivalent; for this reason only downward-going atmospheric muons can be measured [18-20]. For those primary cosmic rays absorbed by the Moon, a deficit in the flux of the secondary muons can be measured, being directly correlated to the position of the Moon in the sky.

The energy threshold for muons detectable at the depth of the ANTARES telescope is about $500 \mathrm{GeV}$ when they are at the sea surface level, most of them with energy above $1 \mathrm{TeV}$. Primaries which are progenitors of such highly energetic muons are practically not affected by the Earth geomagnetic field. This assumption of large rigidity holds also for the secondary muons detected by the ANTARES detector, thus they can be exploited in the study of the Moon shadow without introducing any bias. The smearing of muon direction with respect to the primary cosmic rays due to pion transverse momentum and pion decay is limited by the large Lorentz factor [21]. The analysis presented in this paper covers the data-taking period spanning from 2007 to 2016, corresponding to a total live-time of 3128 days. Figure 1 shows the position of the Moon in the horizontal coordinate system of the detector for such a period. The Moon altitude ranges above the horizon up to about $75^{\circ}$.

The analysis is performed in three steps, described in Sect. 3.1, 3.2 and 3.3. First, quality cuts are defined to reduce the number of candidate atmospheric muon events to a sample which provides the best sensitivity for this search. The second part concerns the estimation of the telescope angular resolution for atmospheric muons by studying the monodimensional profile of the Moon shadow. In the third part, the pointing precision is determined evaluating a possible shift of the measured direction of the Moon with respect to the nominal values provided by astronomical libraries [24].

\subsection{Optimisation of quality cuts}

A dedicated Monte Carlo (MC) production is used to optimize the selection criteria applied to the reconstructed muon tracks. The MC generation of the atmospheric muon sample is performed with the MUPAGE code [25], which was proved to reliably reproduce this background source in several ANTARES analyses, such as reported in [26-28] . MUPAGE implements parametric formulas for the flux, the radial distribution, the multiplicity and the energy spectrum of muons at a given depth, allowing for a fast production of both single and bundle muon events. Muons are generated on the surface of a cylinder-shaped volume of water, $650 \mathrm{~m}$ high, with a radius of $290 \mathrm{~m}$, containing the detector. This volume is larger than the instrumented volume and corresponds to the region in which muons can produce detectable signals. The generation of the MC sample is subdivided in different batches corresponding to the periods of data-taking, 

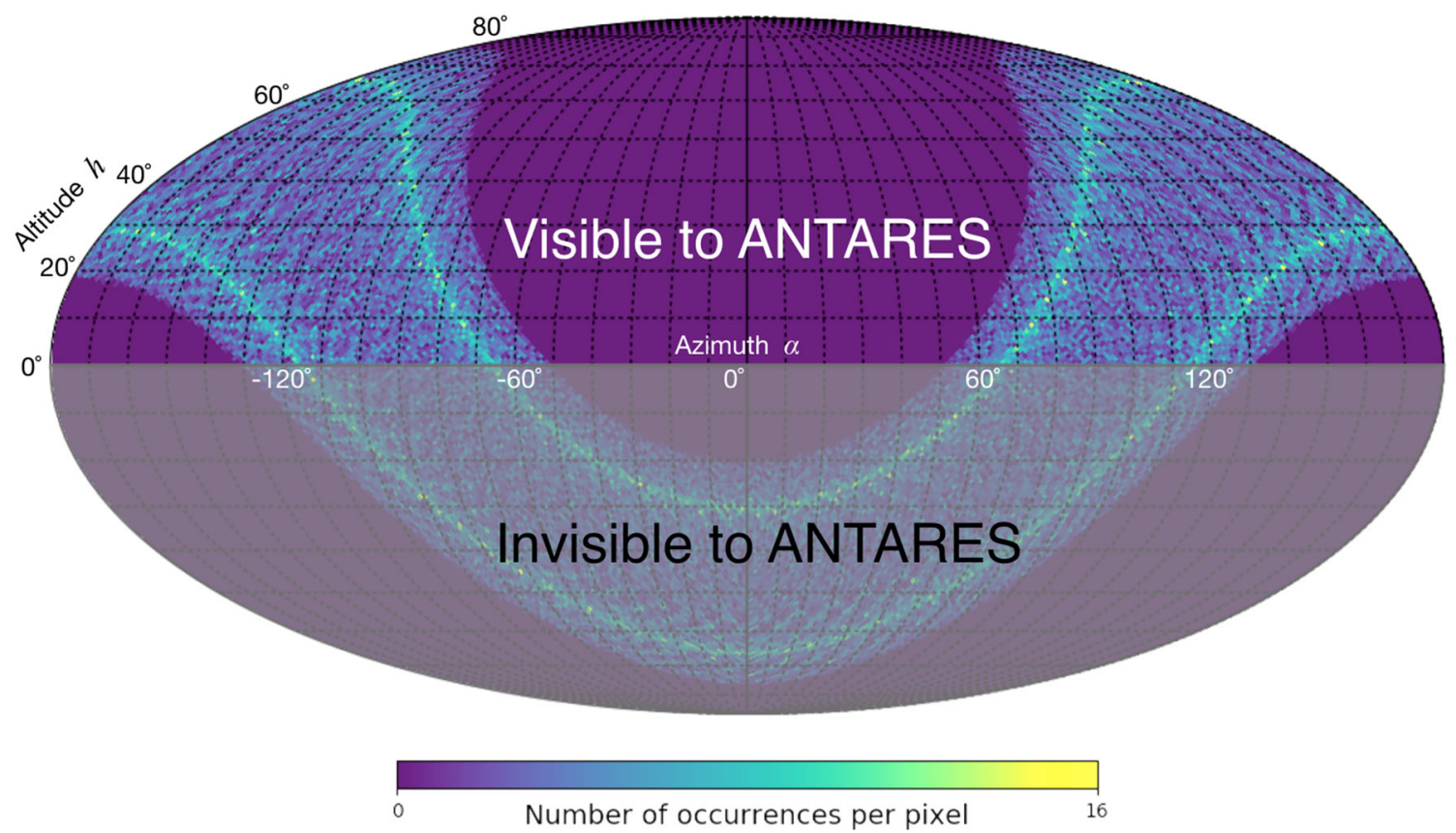

Fig. 1 The visible and invisible sectors of the position of the Moon for ANTARES with respect to the detector horizontal coordinate system. The occurrences of the Moon position are computed at each hour in the period 2007-2016 with the library SkyField [22]. The map is arranged

referred to as runs. The simulation reproduces the effective data taking conditions of the ANTARES detector, which can vary on a run-by-run basis [29]. The simulation includes the generation of Cherenkov light stimulated by the muon and its propagation up to the PMTs on the basis of the measured characteristics of light propagation [30]. Optical background, caused by bioluminescence and radioactive isotopes (mainly ${ }^{40} \mathrm{~K}$ ) present in sea water, is also added according to the measured rate. This technique allows to correlate the actual time of each run to the position of the Moon in the sky. In particular, it is possible to assign an absolute time-stamp, generated randomly within the period of each considered run, to each MC event reconstructed as a downward-going muon. A detailed production compliant with the actual live time is used to generate, reconstruct and select the MC sample of events within the restricted area of $10^{\circ}$ around the nominal position of the Moon at the time of each event. In order to evaluate the contamination of mis-reconstructed events in the proximity of the Moon, a smaller MC sample, with $1 / 3$ of the actual live time, is generated over the whole visible sky.

The detector response is then simulated taking into account the main features of the PMTs and of the electronics $[31,32]$. Finally, the PMT signals are processed to reconstruct the atmospheric muon tracks with the standard ANTARES algorithm for track-like events. This is a robust track-fitting procedure based on a likelihood maximisation [17]. Figures of merit are determined by means of two quality parameters: $\Lambda$, which varies linearly with the logarithm of the recon- according to the Mollweide equal-area view obtained with the use of the HEALPIX package [23], setting the parameter NSIDE $=64$ (i.e. 49152 pixels)

structed track likelihood, and $\beta$, the angular error associated to the reconstructed direction.

Two different MC simulation sets are prepared: the sample $S_{1}$ considering the shadowing effect of the Moon and the sample $S_{0}$ without this effect. In the sample $S_{1}$, the Moon shadow is obtained by removing the muons generated within the Moon disk, assuming a radius of $0.26^{\circ}$. The information from all the considered simulated runs is combined to obtain statistical evidence of the Moon shadow. For each of the two MC samples, $S_{1}$ and $S_{0}$, a one dimensional histogram is built with the distribution of events as a function of the angular distance $\delta$ with respect to the Moon, up to $10^{\circ}$. Such a histogram is subdivided into 25 bins, each one sized $\Delta \delta=0.4^{\circ}$ and corresponding to an annulus of increasing radius centered on the Moon. The content of each bin is normalised to the corresponding annulus area, resulting in an event density.

The cuts on the quality parameters $\Lambda$ and $\beta$ are chosen to achieve the best sensitivity for the Moon shadow detection. The approach of the hypothesis test is used: the null hypothesis $H_{0}$ relates to the case of atmospheric muons without the Moon shadow, while the alternative hypothesis $H_{1}$ corresponds to the presence of the Moon. The used test statistic is defined as $\lambda=-2 \log \frac{L_{H_{1}}}{L_{H_{0}}}$, with $L_{H_{0}}$ and $L_{H_{1}}$ the likelihoods obtained under the $H_{0}$ and $H_{1}$ hypotheses. Assuming that the event population in each bin follows a Poisson probability distribution, using the $\chi^{2}$ definition in [33], the chosen 


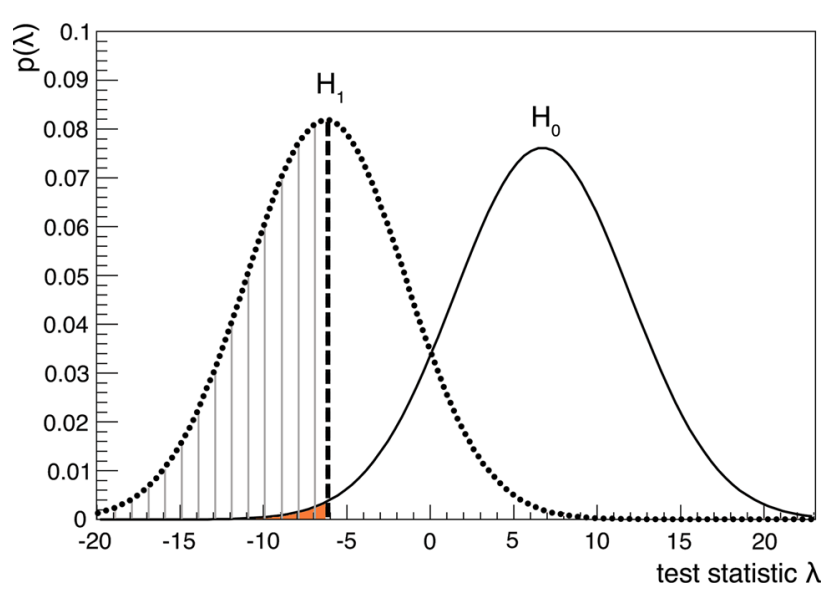

Fig. 2 The test statistics $\lambda$ distribution for the "Moon shadow" hypothesis $H_{1}$ (dotted curve) and the "no Moon shadow" hypothesis $H_{0}$ (smooth curve). The dashed area corresponds to the $50 \%$ of the pseudoexperiments where the Moon shadow hypothesis is correctly identified. The shaded area quantifies the expected median significance (here $3.4 \sigma$ ) to observe the Moon shadow

test statistic can be conveniently written as:

$$
\begin{aligned}
& \lambda=\chi_{H_{1}}^{2}-\chi_{H_{0}}^{2} \\
& \text { with } \\
& \chi_{H}^{2}=2 \sum_{i=1}^{N_{\text {bin }}}\left[N_{i, H}-n_{i}+n_{i} \ln \frac{n_{i}}{N_{i, H}}\right],
\end{aligned}
$$

where $n_{i}$ stands for the measurement in the $i$-th bin to be compared with the expectations $N_{i, H}$ under the $H_{0}$ and $H_{1}$ hypotheses. The following reduced expression for $\lambda$ is used:

$\lambda=2 \sum_{i=1}^{N_{\text {bin }}}\left[\mu_{i}-v_{i}+n_{i} \ln \frac{v_{i}}{\mu_{i}}\right]$,

where for simplicity the expected counts $N_{i, H_{0}}$ and $N_{i, H_{1}}$ are renamed as $v_{i}$ and $\mu_{i}$, respectively. The two possible distributions of $\lambda, f\left(\lambda \mid H_{0}\right)$ and $f\left(\lambda \mid H_{1}\right)$, valid separately under the hypotheses $H_{0}$ and $H_{1}$, respectively, are obtained by means of pseudo-experiments (PEs). The number of events in the $i$-th bin $n_{i}$ is determined by extracting $10^{6}$ random values generated according to a Poisson distribution with expectation values equal to $v_{i}$ and $\mu_{i}$.

Several hypothesis tests are performed assuming different selection criteria for $\Lambda$ and $\beta$. For each set of values, the distributions $f\left(\lambda \mid H_{0}\right)$ and $f\left(\lambda \mid H_{1}\right)$ are compared. The median of $f\left(\lambda \mid H_{1}\right)$ is taken as the critical value for $\lambda$, i.e. as the threshold to separate the two hypothesis. The set of best cut values of $\Lambda$ and $\beta$ corresponds to that for which the two $f(\lambda \mid H)$ distributions have the minimal overlap. Figure 2 shows the distribution $f\left(\lambda \mid H_{0}\right)$ (black curve) and $f\left(\lambda \mid H_{1}\right)$ (red curve) for the optimised quality cuts $\Lambda_{\text {cut }}=-5.9$, $\beta_{\text {cut }}=0.8^{\circ}$, and the critical value is $\lambda=-6.15$. The dashed area below $f\left(\lambda \mid H_{1}\right)$ represents the fraction of PEs where the

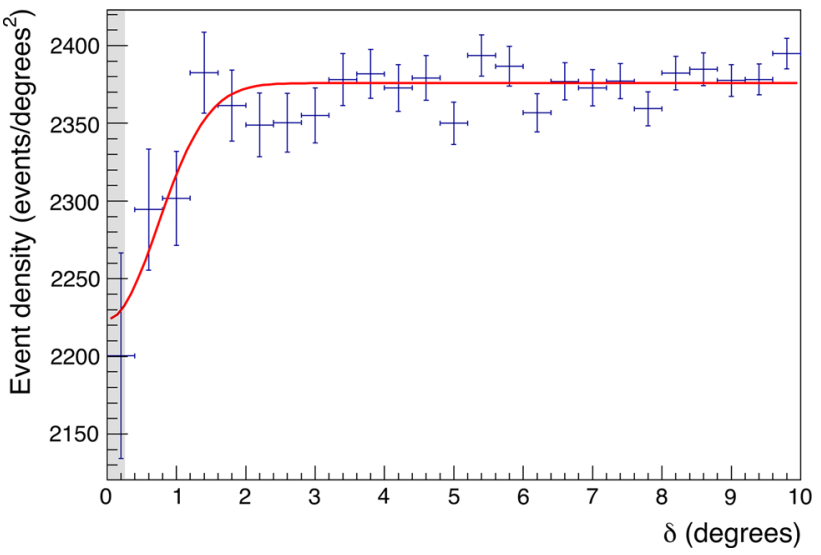

Fig. 3 Measured muon event density as a function of the angular distance $\delta$ from the Moon. Data histogram is shown with statistical errors; the smooth line is the best fit according to Eq. (4); the shaded area corresponds to the apparent radius of the Moon $\left(0.26^{\circ}\right)$

Moon shadow hypothesis is correctly identified; the filledcoloured area below $f\left(\lambda \mid H_{0}\right)$ corresponds to a $p$-value equal to $3.6 \times 10^{-4}$, or equivalently $3.4 \sigma$. This is the expected median significance of the Moon shadow effect with the MC data set.

\subsection{Deficit significance and angular resolution}

The optimized quality cuts reported above are applied to the data sample collected in the period 2007-2016, selecting $9.6 \times 10^{5}$ events out of the pre-cuts collection about three times larger. Figure 3 shows the resulting distribution for the muon density as a function of $\delta$ in the range of $0^{\circ} \leq \delta \leq 10^{\circ}$ with bin size of $\Delta \delta=0.4^{\circ}$. A clear deficit of events is evident in the region around the Moon position $\left(\delta<1.2^{\circ}\right)$.

For the estimation of the angular resolution, the Moon shadowing effect is assumed to follow a Gaussian distribution with standard deviation $\sigma_{r e s}$, which corresponds to the detector angular resolution itself. This is motivated by the fact that the apparent size of the Moon in the sky is sufficiently small compared to the expected value of the detector angular resolution, affecting the estimation by less than a few percents. A similar approach has already been followed by $[5,8,34]$. The number of expected events is evaluated by fitting the distribution in Fig. 3 with the following function [6]:

$\frac{d n}{d \delta^{2}}=k\left(1-\frac{R_{M o o n}^{2}}{2 \sigma_{r e s}^{2}} e^{-\frac{\delta^{2}}{2 \sigma_{\text {res }}^{2}}}\right)$

The two free parameters are $k$, the average muon event density in the $H_{0}$ scenario, and $\sigma_{\text {res }}$. The Moon radius $R_{M o o n}$ is fixed to $0.26^{\circ}$.

The angular resolution for downward-going atmospheric muons resulting from the fit is $\sigma_{\text {res }}=0.73^{\circ} \pm 0.14^{\circ}$, with 
the fitted value of $k=2376 \pm 3$ events per square degrees. The goodness of the fit is found to be $\chi^{2} /$ dof $=23.5 / 23$.

The significance of the shadowing is evaluated using a $\chi^{2}$ test comparing the measured event density with the flat distribution $\frac{d n}{d \delta^{2}}=k$. Such $\chi^{2}$ test leads to a $p$-value equal to $4.3 \times 10^{-4}$ corresponding to a significance of the Moon shadow effect of $3.3 \sigma$. This value is compatible with the expected significance of the Monte Carlo previously described.

\subsection{Absolute pointing}

The procedure for evaluating the pointing accuracy of the Moon shadow is partially inspired by [5]; it is based on determining the statistical significance of the selected data set under the assumption of the Moon in a given direction. All possible placements are considered within a field of view $(\mathrm{FoV})$ centered on the nominal position of the Moon. This work differs from [5] in the way the significance of the results is evaluated.

The event distribution of the detected muons, compliant to the determined quality cuts, is represented as function of $x=\left(\alpha_{\mu}-\alpha_{\text {Moon }}\right) \times \cos \left(h_{\mu}\right)$ and $y=h_{\mu}-h_{\text {Moon }}$; here $\left(\alpha_{\mu}, h_{\mu}\right)$ and $\left(\alpha_{\text {Moon }}, h_{\text {Moon }}\right)$ are the horizontal coordinates of the track and the Moon, respectively, at the time of the event.

The FoV is limited in both $x$ and $y$ within the range $\left[-10^{\circ}, 10^{\circ}\right]$, and it is subdivided in a grid of $0.2^{\circ} \times 0.2^{\circ}$ squared bins. The used test statistic is again $\lambda$ as reported in Eq. (3), but now the sum is evaluated on all $100 \times 100$ square bins.

The expectations under $H_{0}$ are obtained parameterising the event distribution of the measured atmospheric muons which fall in the FoV relative to the position of the Moon four hours before the timestamp of each event. The parameterisation is done with a second degree polynomial of the form:

$p_{2}\left(x, y, \mathbf{k} \mid H_{0}\right)=k_{0}+k_{1} x+k_{2} x^{2}+k_{3} y+k_{4} y^{2}$,

with the fitted parameter array $\mathbf{k} \equiv\{93.6 \pm 1.8,0.19 \pm 0.16$, $\left.(-8.2 \pm 3.1) \times 10^{-3}, 3.98 \pm 0.17,(5.60 \pm 0.32) \times 10^{-2}\right\}$. The goodness of the fit is $\chi^{2} /$ dof $=9993 / 9995$ for this set of values $\mathbf{k}$, corresponding to a $p$-value $\approx 0.5$; it validates the modelling of the event distribution in the absence of the Moon provided by Eq. (5).

Figures $4 \mathrm{a}, \mathrm{b}$ represent the projection of the event distribution in the FoV onto the $x$ and $y$ axes in the absence of the Moon shadow, also called marginal distributions. The marginal distribution for $x$ is almost flat, compliant with the expected lack of any significant structure in the atmospheric muon flux along the azimuth. On the contrary, the marginal distribution for $y$ shows an almost linear ramping

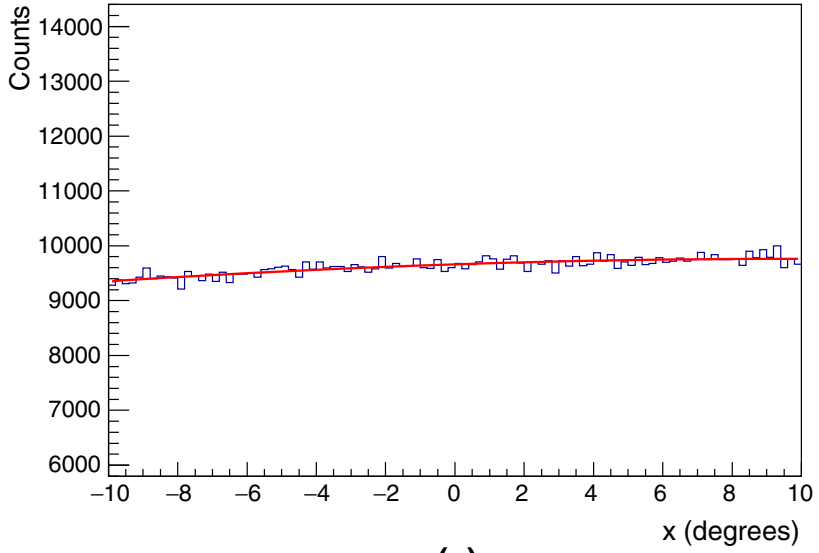

(a)

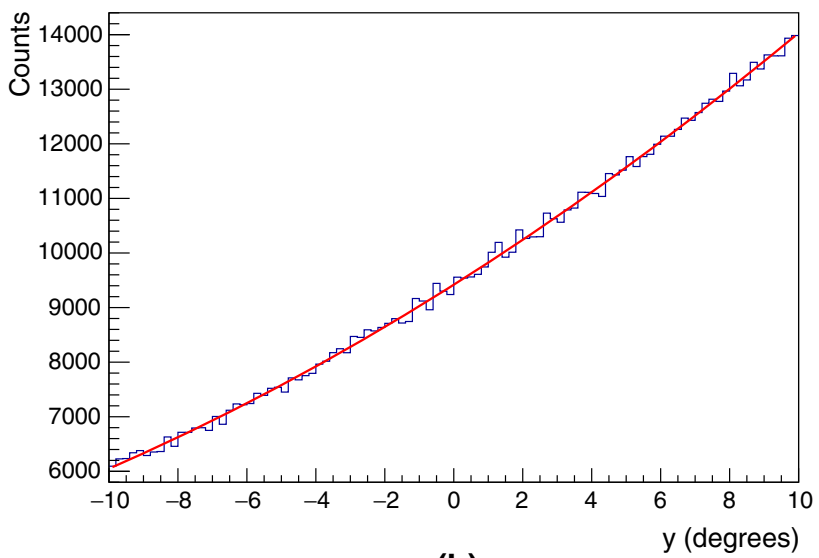

(b)

Fig. 4 Projection of the measured 2-D event distributions, in absence of the Moon shadow $\left(H_{0}\right)$, for the field of view coordinates $x=\left(\alpha_{\mu}-\right.$ $\left.\alpha_{\text {Moon }}\right) \times \cos h_{\mu}$ and $y=h_{\mu}-h_{\text {Moon }}$

which reflects the enhancement of the muon flux with the altitude.

The expectations under $H_{1}$ are then obtained by subtracting from $p_{2}\left(x, y, \mathbf{k} \mid H_{0}\right)$ a bi-dimensional Gaussian point spread function:

$G(x, y, \theta)=\frac{A}{2 \pi \sigma_{r e s}^{2}} e^{-\frac{\left(x-x_{s}\right)^{2}+\left(y-y_{s}\right)^{2}}{2 \sigma_{r e s}^{2}}}$.

In Eq. (6) the same spread is assumed in both dimensions, so that $\sigma_{x}=\sigma_{y} \equiv \sigma_{\text {res }}$. The $\sigma_{\text {res }}$ is fixed to value of the angular resolution found in the previous sub-section. The array of free parameters $\theta$ is composed of the amplitude of the Moon deficit $A$ and the assumed position of the Moon $\left(x_{s}, y_{s}\right)$ in the FoV.

For each bin in the FoV, the value of the test statistic $\lambda$ is minimised finding the best estimation of $A$. The smallest value $\lambda_{\min }$ is found equal to -17.05 , for the fitted deficit amplitude $A_{\min }=20 \pm 5$, in the bin with center in $x=0.5^{\circ}$ and $y=0.1^{\circ}$. Such coordinates are taken as the best esti- 


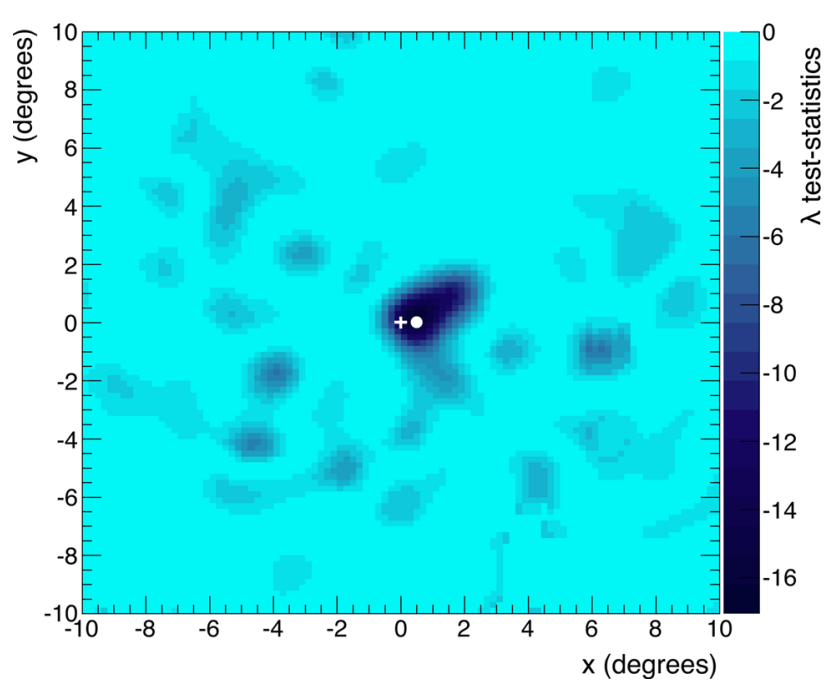

Fig. 5 Measured distribution of the test statistic $\lambda$ from Eq. (3) in the field of view around the Moon nominal position $O \equiv\left(0^{\circ}, 0^{\circ}\right)$, indicated by a white cross. The white dot refers to the coordinates $\left(0.5^{\circ}, 0.1^{\circ}\right)$ where the test statistics reaches the minimum $\left(\lambda_{\min }=-17.05\right)$

mation of the position of the Moon. The test statistic $\lambda_{O}$ in the nominal position $O \equiv\left(0^{\circ}, 0^{\circ}\right)$ is found equal to -13.37 for the corresponding amplitude $A_{O}=19 \pm 5$. At each bin, $-\lambda$ follows the distribution of a central $\chi^{2}$ with one degree of freedom, assuming $H_{0}$ as true. This allows to estimate the discrepancy of the measured data from the assumption of the absence of the Moon. Considering $\lambda_{O}$, a $p$-value of $2.6 \times 10^{-4}$ is obtained, which corresponds to a significance of $3.5 \sigma$, in agreement with what is reported in the above Sect. 3.2. Figure 5 shows the $\lambda$ distribution in the FoV. It can be interpreted as a bi-dimensional profile-likelihood, with $A$ treat-ed as the nuisance parameter. The interval corresponding to a desired confidence level $(C L)$ is obtained for $\lambda \leq \lambda_{\text {cut }}=\lambda_{\text {min }}+Q$, where $Q$ is the quantile accounting for two degrees of freedom and confidence level $C L$ [35].

An additional strategy is used to cross-check the confidence intervals found with the method reported above. This is done by exploiting the PE technique. In each bin of the FoV, a reference number of events $\left\{n_{i}\right\}_{\text {ref }}$ is computed using the superposition of Eqs. (5) and (6). For this purpose the Moon is assumed to be in $O, \sigma_{\text {res }}=0.73^{\circ}$ and $A=A_{O}$. For each PE, a corresponding data set $\left\{n_{i}\right\}_{P E}$ is extracted as Possionian fluctuations of the reference set $\left\{n_{i}\right\}_{\text {ref }}$. Using $10^{5} \mathrm{PEs}$, the distribution of the best value of $\lambda_{j}$ is determined at the $j$-th bin of the FoV.

For each $\lambda_{j}$ distribution, the range $\left(-\infty, \lambda_{j}^{C L}\right]$ is considered, where $\lambda_{j}^{C L}$ is the value of $\lambda_{j}$ such that its cumulative distribution is $F\left(\lambda_{j}^{C L}\right)=C L$; the $j$-th bin is included into the confidence interval if $\lambda_{j}^{m} \leq \lambda_{j}^{C L}$.

Figure 6 shows the estimation of the confidence regions for $C L \equiv\{68.27 \%, 95.45 \%, 99.73 \%\}$ using both the meth-

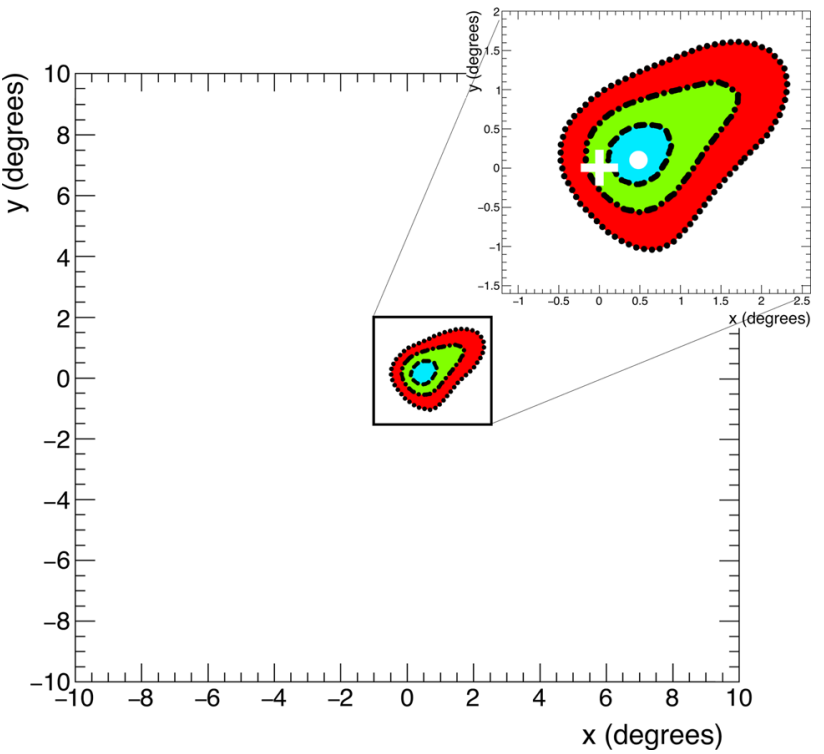

Fig. 6 Contour plots corresponding to different confidence levels (cyan/dashed: 68.27\%; green/dot-dashed: 95.45\%; red/dotted: $99.73 \%$ ), computed with the two methods described in the text. In the zoom, the dot represents the position in the FoV where $\lambda_{\min }=-17.05$. The cross indicates the nominal position of the Moon

ods explained above. The contours found with the first and the second methods are indicated by colours and lines, respectively. The contour plots of the two approaches are in excellent agreement.

The statistical significance of the apparent shift with respect to the Moon nominal position is determined using PEs. The method relies on the probability density function of the test statistics $\Theta=\lambda_{O}-\lambda_{\min }$, with $\lambda_{O}$ and $\lambda_{\min }$ defined as before. The $\Theta$ test statistic is interpreted as a profile likelihood whose distribution asymptotically tends to that one of a $\chi^{2}$ with two degrees of freedom.

In Fig. 7 the normalised distribution of the $\Theta$ test statistic is shown, where the measured value of the test statistic $\Theta_{\text {meas }}=3.68$ is indicated for reference by the red-dashed line. Integrating the $\Theta$ distribution for values larger than $\Theta_{\text {meas }}$, a $p$-value $=0.23$ is obtained, corresponding to a significance of $1.2 \sigma$. This indicates that the shift is compatible with a statistical fluctuation.

\section{Analysis of data collected with a surface array}

The pointing performance of the ANTARES telescope is cross-checked in a completely independent way, exploiting the measurements made with a surface array detector. The device was temporarily onboard of a ship circulating around the position of the telescope, synchronised to a GPS reference. The surface array was composed of a set of 15 liquid scintillator detection units, designed for the measurement of 


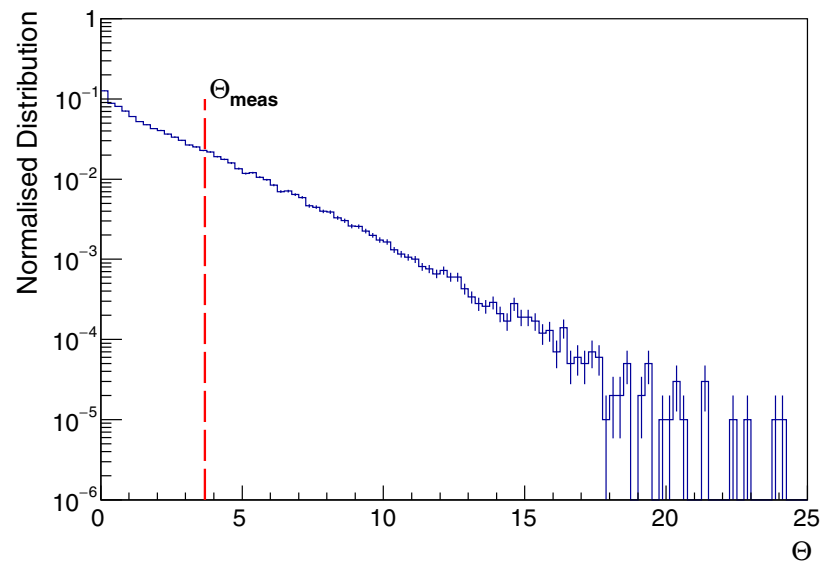

Fig. 7 Distribution of the $\Theta=\lambda_{O}-\lambda_{\min }$ test statistics, obtained with $10^{5}$ pseudo-experiments assuming the Moon in the nominal position $O$. The $23 \%$ of the pseudo-experiments has a test statistic $\Theta$ larger than the measured value $\Theta_{\text {meas }}=3.68$, indicated for reference by the red-dashed line

atmospheric showers, placed over an area of about $50 \mathrm{~m}$ $\times 14 \mathrm{~m}$ on the ship deck. Each scintillator unit included a polyethylene-aluminium box filled with linear alkylbenzene doped with wavelength shifters. The scintillation light was detected using 2" PMTs, one per unit. Each scintillator unit had a single rate of around $100 \mathrm{~Hz}$. The pointing accuracy of the ANTARES detector is inferred by combining the data from the surface array and the undersea telescope.

Two different sea campaigns were performed: a first campaign of seven days in 2011 and a second campaign of six days in 2012. Given the area covered by the ship routing above the ANTARES telescope, the range of the muon zenith $\theta$ is limited to $2^{\circ} \leq \theta \leq 27^{\circ}$. Figure 8 shows the recorded positions on the sea surface of the ship during these two periods.

The shower array is used to trigger the possible timecorrelations with the ANTARES events. The typical trigger rate of the surface array is around $1 \mathrm{~Hz}$ requiring coincidences in at least 3 detection units in a $650 \mathrm{~ns}$ time window. The rate of reconstructed muons is $\sim 0.25 \mathrm{~Hz}$ when applying cuts on the quality parameters $\Lambda \geq-6$ and $\beta \leq 0.6^{\circ}$. The coincidence time-window between the surface array and the underwater telescope is set to $10 \mu \mathrm{s}$. The rate of coincidences is about 40 per day, with an expected rate of random coincidences of about 0.2 events per day.

The direction of the atmospheric shower is estimated by correlating the GPS position of the ship with the ANTARES barycentre. An uncertainty of $25 \mathrm{~m}$, about one half of the ship deck hosting the shower array, is assumed for the shower position detected by the array. An error of $70 \mathrm{~m}$ is considered for the possible displacement of the muon/muon bundle axis inside the detector volume. Considering only the ship routes

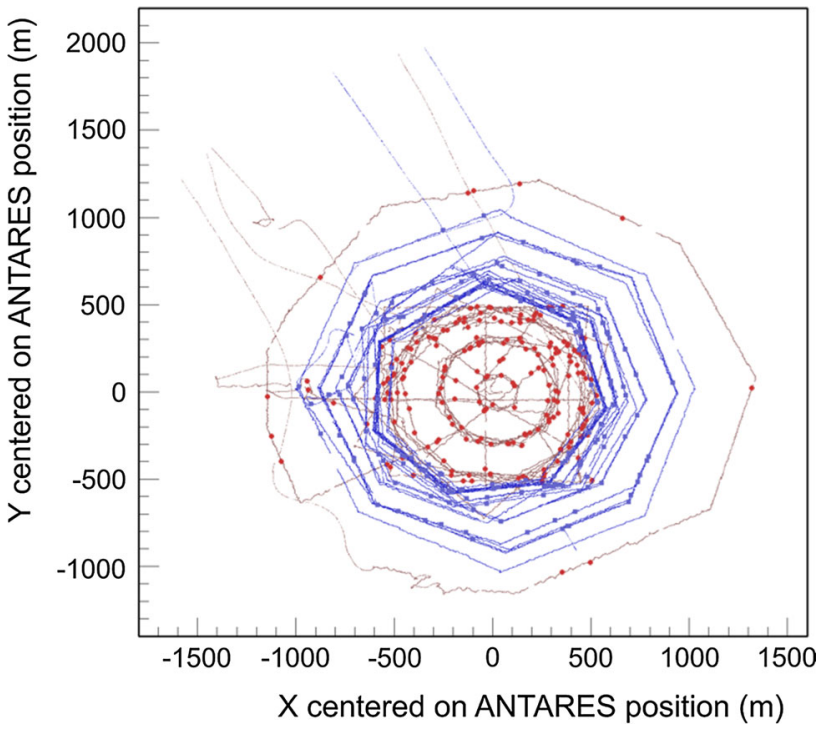

Fig. 8 Ship route in the 2011 (red) and 2012 (blue) campaigns around the center of the ANTARES detector
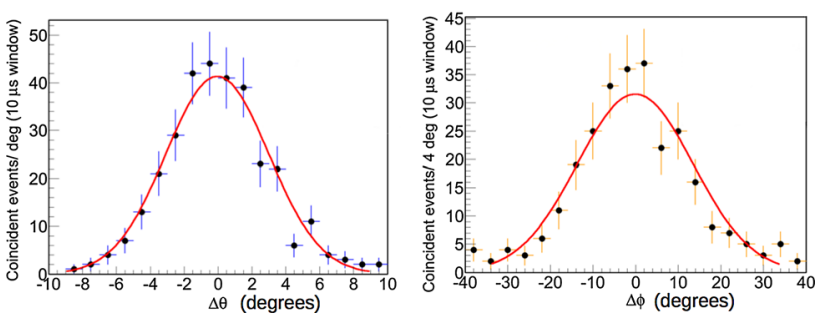

Fig. 9 Left: the difference between the zenith angles of the shower axis (determined as the direction of the ship with respect to the ANTARES location) and of the reconstructed muon underwater. Right: the same for the azimuth angles. Fit results: $\Delta \theta_{\text {mean }}=-0.07^{\circ} \pm 0.22^{\circ} ; \Delta \phi_{\text {mean }}=$ $-0.5^{\circ} \pm 0.8^{\circ}$

with radius larger than $500 \mathrm{~m}$, the estimated resolutions are $\sim 3^{\circ}$ in zenith and $\sim 8^{\circ}$ in azimuth.

The results of the two campaigns are shown in Fig. 9: the represented $\Delta \theta$ (left) and $\Delta \phi$ (right) are the differences between the directions of the shower axis and the reconstructed muon underwater.

According to the Gaussian fit of the two distributions in Fig. 9, the absolute pointing appears to be consistent with the nominal expectations, i.e. with a null systematic shift in both zenith and azimuth within the errors (see caption of Fig. 9). The large uncertainty in the azimuth estimation is due to the low zenith angle tested during the campaigns, as can be derived from Fig. 8. The results of the surface array analysis are in good agreement with the pointing performance found with the Moon shadow analysis. 


\section{Conclusions}

This paper describes the estimations of the pointing performance of the ANTARES telescope using the Moon shadow effect and a dedicated surface array.

The selected events from the data recorded in the 20072016 period with altitude angles $0^{\circ} \leq h \leq 75^{\circ}$, allowed the identification of the Moon shadow with $3.5 \sigma$ statistical significance. The corresponding detector angular resolution for downward-going atmospheric muons is $0.73^{\circ} \pm 0.14^{\circ}$.

The pointing accuracy of the detector is consistent with the expectations.

Acknowledgements The authors acknowledge the financial support of the funding agencies: Centre National de la Recherche Scientifique (CNRS), Commissariat à l'énergie atomique et aux énergies alternatives (CEA), Commission Européenne (FEDER fund and Marie Curie Program), Institut Universitaire de France (IUF), IdEx program and UnivEarthS Labex program at Sorbonne Paris Cité (ANR-10LABX-0023 and ANR-11-IDEX-0005-02), Labex OCEVU (ANR-11LABX-0060) and the A*MIDEX project (ANR-11-IDEX-0001-02), Région Île-de-France (DIM-ACAV), Région Alsace (contrat CPER), Région Provence-Alpes-Côte d'Azur, Département du Var and Ville de La Seyne-sur-Mer, France; Bundesministerium für Bildung und Forschung (BMBF), Germany; Istituto Naziona-le di Fisica Nucleare (INFN), Italy; Nederlandse organisatie voor Wetenschappelijk Onderzoek (NWO), the Netherlands; Council of the President of the Russian Federation for young scientists and leading scientific schools supporting grants, Russia; National Authority for Scientific Research (ANCS), Romania; Ministerio de Economía y Competitividad (MINE-CO): Plan Estatal de Investigación (refs. FPA2015-65150-C3-1-P, -2-P and -3-P, (MINECO/FEDER)), Severo Ochoa Centre of Excellence and MultiDark Consolider (MINECO), and Prometeo and Grisolía programs (Generalitat Valencia-na), Spain; Ministry of Higher Education, Scientific Research and Professional Training, Morocco. We also acknowledge the technical support of Ifremer, AIM and Foselev Marine for the sea operation and the CC-IN2P3 for the computing facilities.

Open Access This article is distributed under the terms of the Creative Commons Attribution 4.0 International License (http://creativecomm ons.org/licenses/by/4.0/), which permits unrestricted use, distribution, and reproduction in any medium, provided you give appropriate credit to the original author(s) and the source, provide a link to the Creative Commons license, and indicate if changes were made.

Funded by SCOAP ${ }^{3}$.

\section{References}

1. M. G. Aartsen et al. (IceCube Collaboration), Science 342, 1242856 (2013)

2. M. G. Aartsen et al. (IceCube Collaboration), Journal of Instrumentation 12(3), P03012 (2017)

3. M. G. Aartsen et al. (IceCube Collaboration), Phys. Rev. Lett. 113, 101101 (2017)

4. M. Ageron et al. (ANTARES Collaboration), Nucl. Instrum. Meth. A656, 11-38 (2011)
5. M. Ambrosio et al. (MACRO Collaboration), Phys. Rev. D59, 012003 (1999)

6. J. H. Cobb et al. (Soudan 2 Collaboration), Phys. Rev. D61, 092002 (2000)

7. P. Achard et al., Astropart. Phys. 23(4), 411-434 (2005)

8. M. G. Aartsen et al. (IceCube Collaboration), Phys. Rev. D89(10), $102004(2014)$

9. D. E. Alexandreas et al. (CYGNUS Collaboration), Phys. Rev. D43, 1735-1738 (1991)

10. M. Amenomori et al. (Tibet AS gamma Collaboration), Phys. Rev. D47, 2675-2681 (1993)

11. A. Borione et al., Phys. Rev. D 49, 1171-1177 (1994)

12. B. Bartoli et al. (ARGO-YBJ Collaboration), Phys. Rev. D85, 022002 (2012)

13. A. U. Abeysekara et al. (HAWC Collaboration), Phys. Rev. D97(10), 102005 (2018)

14. J. A. Aguilar et al. (ANTARES Collaboration), Astropart. Phys. 34, 539-549 (2011)

15. S. Adrian-Martinez et al. (ANTARES Collaboration), JINST 7, T08002 (2012)

16. J. A. Aguilar et al. (ANTARES Collaboration), Astropart. Phys. 34(9), 652-662 (2011)

17. S. Adrian-Martinez et al. (ANTARES Collaboration), JCAP 1303, 006 (2013)

18. J. A. Aguilar et al. (ANTARES Collaboration), Astropart. Phys. 34(3), 179-184 (2010)

19. J. A. Aguilar et al. (ANTARES Collaboration), Astropart. Phys. 33(2), 86-90 (2010)

20. S. Adrian-Martinez et al. (ANTARES Collaboration), Astropart. Phys. 78(C), 43-51 (2016)

21. C. Distefano (ANTARES Collaboration), Nucl. Instrum. Meth A626-627, S223-S225 (2011)

22. B. Rhodes, in SkyField, Elegant Astronomy for Python, http:// rhodesmill.org/skyfield/. Accessed 31 July 2018

23. K.M. Górski, E. Hivon, A.J. Banday, B.D. Wandelt, F.K. Hansen, M. Reinecke, M. Bartelmann, Astrophys. J. 622, 759-771 (2005)

24. J. H. Meeus, in Astronomical Algorithms., ed. by Willmann-Bell, Incorporated (1991)

25. G. Carminati, A. Margiotta, M. Spurio, Comput. Phys. Commun. 179, 915-923 (2008)

26. A. Albert et al. (ANTARES Collaboration), JHEP 07, 54 (2017)

27. A. Albert et al. (ANTARES Collaboration), Phys. Rev. D96, 082001 (2017)

28. J.A. Aguilar et al. (ANTARES Collaboration), Astropart. Phys. 34, 179-184 (2010)

29. L.A. Fusco, A. Margiotta, EPJ Web Conf. 116, 02002 (2016)

30. H. Yepes-Ramirez (ANTARES Collaboration), Nucl. Instrum. Meth A725, 203-206 (2013)

31. J. Brunner (ANTARES Collaboration), in Proceedings of the VLVnuT Workshop, Amsterdam, Netherlands, October 5-8, 2003, ed. by E. de Wolf (Amsterdam: NIKHEF), http://www.vlvnt.nl/ proceedings/

32. A. Margiotta (ANTARES Collaboration), Nucl. Instrum. Meth. A725, 98-101 (2013)

33. S. Baker, R.D. Cousins, Nucl. Instrum. Meth. 221, 437-442 (1984)

34. P. Adamson et al. (MINOS Collaboration), Astropart. Phys. 34(6), 457-466 (2011)

35. G. Cowan, Statistical Data Analysis (Clarendon Press, Oxford, 1998) 